\title{
EVALUACIÓN POBLACIONAL Y USO SOSTENIBLE DE ANIMALES DE CAZA POR COMUNIDADES INDÍGENAS EN EL ÁREA DE CONSERVACIÓN REGIONAL AMPIYACU APAYACU, NORESTE DE LA AMAZONÍA PERUANA
}

\author{
Pablo E. PUERTAS ${ }^{1}$, Alejandro PINEDO ${ }^{2}$, Sandro SOPLÍN ${ }^{3}$, Miguel ANTÚNEZ ${ }^{4}$, Luis LÓPEZ ${ }^{3}$, Jhonatan \\ CARO $^{3}$ Liz CHICAJE ${ }^{5}$ Rolando PANDURO ${ }^{6}$ René VASQUEZ ${ }^{1}$ José Luis FLORES \\ 1 Instituto del Bien Común (IBC), Calle Nauta 334, Iquitos - Perú. E-mail: pepuertas@gmail.com \\ 2 Dirección Ejecutiva de Conservación y Diversidad Biológica - Autoridad Regional Ambiental de Loreto (ARA) del \\ Gobierno Regional de Loreto (Gore Loreto). \\ 3 Universidad Nacional de la Amazonía Peruana (UNAP). \\ 4 Fundación Latinoamericana para el Trópico Amazónico (FUNDAMAZONIA). \\ 5 Federación de Comunidades Nativas del Ampiyacu (FECONA). \\ 6 Comité de Gestión del Área de Conservación Regional Ampiyacu Apayacu.
}

\section{RESUMEN}

Este estudio presenta los resultados de la evaluación poblacional de fauna silvestre y el conocimiento de las especies sujetas a caza sostenible por comunidades pertenecientes a la Federación de Comunidades Nativas del Ampiyacu (FECONA) durante el 2016 en la zona de aprovechamiento directo del Área de Conservación Regional Ampiyacu Apayacu (ACRAA). Las comunidades locales participaron en la evaluación bajo el liderazgo de la Dirección Ejecutiva de Conservación y Diversidad Biológica del Gobierno Regional de Loreto. Aplicamos las metodologías de registros de caza, diálogos interactivos y censos de fauna silvestre. El método de cosecha unificado fue empleado para realizar el análisis de sostenibilidad de la caza. En total, fueron seis las especies más preferidas por la población local, que soportan la presión de caza y se encuentran por debajo del límite de cosecha sostenible. Estas especies fueron: Pecari tajacu "sajino", Tayassu pecari "huangana", Mazama americana "venado colorado", M. nemorivaga "venado gris", Cuniculus paca "majás" y Dasyprocta fuliginosa "añuje". La información proporcionada en este artículo, está siendo utilizada por el Gobierno Regional de Loreto para definir las tasas de aprovechamiento comercial a pequeña escala de las especies que soportan la presión de caza, hasta que se elabore un plan de manejo. Esta experiencia piloto es única dada para las áreas de conservación regional en la amazonía de Loreto, cuyas lecciones aprendidas indudablemente serán aplicadas con las adaptaciones del caso a otras áreas de conservación regional.

PALABRAS CLAVE: Área de Conservación Regional Ampiyacu Apayacu, carne de animales de caza, pueblos indígenas, animales silvestres, caza.

\section{EVALUATION OF THE POPULATION AND SUSTAINABLE USE OF WILDLIFE BY INDIGENOUS COMMUNITIES IN THE AMPIYACU APAYACU REGIONAL CONSERVATION AREA, NORTHEAST OF THE PERUVIAN AMAZONIA}

\begin{abstract}
This study presents the results of the assessment of wildlife population and knowledge of species subject to sustainable hunting by communities belonging to the Federation of Native Communities of the Ampiyacu (FECONA) during 2016 in the direct use zone of the Ampiyacu Apayacu Regional Conservation Area (AARCA). Local communities participated in the assessment under the leadership of the Executive Directorate for Conservation and Biological Diversity of the Regional Government of Loreto. We applied the methodologies of hunting records, interactive dialogues and wildlife censuses. The unified harvesting method was used to analyze the sustainability of the hunting. In the AARCA, six species were most preferred by the local communities that support the hunting pressure and are below the sustainable harvest limit. These species were: Pecari tajacu "collared peccary", Tayassu pecari "white-lipped peccary", Mazama americana "red brocket deer", M. nemorivaga "gray deer", Cuniculus paca "paca" and Dasyprocta fuliginosa "agouti". This information is being used by the Regional Government to define the rates of small-scale commercial use of the species until a management plan is designed. This pilot experience is unique given to the regional conservation areas in the Amazon of Loreto, whose lessons learned will undoubtedly be applied with the adaptations of the case to other conservation areas.
\end{abstract}

KEYWORDS: Ampiyacu Apayacu Regional Conservation Area, game meat, indigenous peoples, wild animals, hunting 


\section{INTRODUCCIÓN}

El Área de conservación Regional Ampiyacu Apayacu (ACR AA) y su zona de influencia posee uno de los bosques más biodiversos de la Amazonía conteniendo una extraordinaria riqueza de flora y fauna silvestre con más de 1,500 especies de vertebrados y 3,500 especies de plantas, destacando la mayor densidad de tapires del planeta (Pitmann et al. 2004). Por esa razón fundamental, se crea la referida área de conservación a fin de garantizar el acceso a los recursos naturales mediante el uso sostenible de la flora y fauna silvestre, promoviendo su uso sostenible y mejora de la calidad de vida de los habitantes de la zona (Gorel - Procrel, 2013). Implementando en colaboración con las comunidades locales y el Gobierno Regional de Loreto (GORE LORETO), programas a largo plazo para monitorear cosechas de animales usadas como carne de monte y cuya forma de aprovechamiento sea realizada mediante iniciativas de manejo que garanticen el uso sostenible de la fauna silvestre (Pitmann etal., 2004).

La evaluación del uso sostenible de la caza fue realizada usando el modelo simple de cosecha unificado desarrollada por Bodmer (2001) y siguiendo las consideraciones referidas por Robinson \& Bodmer (1999) con el propósito de determinar las especies que están siendo sobre cazadas, así como de evaluar si el nivel de cosecha está siendo riesgosa o segura dependiendo del tamaño de la población en relación al límite máximo de cosecha sostenible estimada.

Existe información valiosa disponible sobre la fauna silvestre existente y que está siendo aprovechada en el ACR AA y su zona de influencia. Estas informaciones se encuentran en el inventario biológico rápido producido por el Field Museum de Chicago (Pitman et al., 2004), en el Plan Maestro 2012 - 2016 (GOREL, 2013), el plan de monitoreo del ACR AA y su zona de influencia (Gore Loreto/Procrel, 2015). Así como de documentos de divulgación comunal - cartillas orientadas a 1) los lineamientos para el uso de recursos naturales (GOREL, 2013) y 2) al control y vigilancia comunal (Gorel/Procrel, 2013). Finalmente, de las informaciones acopiadas por personal profesional del Instituto del Bien Común (IBC) y del GORE LORETO - PROCREL.

Los participantes en las actividades de monitoreo de fauna silvestre, orientada al conocimiento del uso sostenible de aquellas especies prioritarias de caza, fueron de los pueblos originarios Bora, Huitoto, Yaguas y Ocaína pertenecientes a la Federación de Comunidades Nativas del Ampiyacu (FECONA), con el liderazgo de la Jefatura del ACR AA, el Presidente del Comité de Gestión del ACR AA, dirigentes de FECONA, así como de profesores y alumnos de los centros educativos de la cuenca del Ampiyacu. En la actualidad, existe un gran interés por las comunidades locales de continuar conduciendo tales actividades generando información y que contribuya a la elaboración de un plan de manejo de fauna silvestre con aquellas especies cuya cacería está siendo sostenible.

La información presentada en este documento será de gran utilidad en el proceso de implementación de las actividades de monitoreo de fauna silvestre orientada a los animales de caza en el ACR AA con extensión a su zona de influencia, mejorando en la aplicación metodológica y de obtención de la información que garantizará la sostenibilidad de la actividad mediante el auto monitoreo comunal, así como en la elaboración de planes de manejo de fauna silvestre con activa participación de la población local. Indudablemente, la información proporcionada será de gran contribución a efectos de garantizar la seguridad alimentaria y como alternativa de mitigación ante eventos adversos del cambio climático que se viene experimentando cada vez más en la Amazonía peruana de Loreto.

\section{MATERIAL Y MÉTODOS}

\section{ÁREA DE ESTUDIO}

Las actividades de monitoreo de fauna silvestre se realizaron en la cuenca del río Ampiyacu, en las comprensiones del ACRAA. Este río cuenta con una superficie de 2918,72 $\mathrm{Km}^{2}$, está ubicada a la margen izquierda del río Amazonas; en el distrito de Pebas, provincia de Mariscal Ramón Castilla, en el departamento de Loreto. Tiene como tributarios a los ríos Yaguasyacu (con una superficie de 1563.79 $\mathrm{Km}^{2}$ ), Zumún y las quebradas, Sábalo y Airambo, entre otras quebradas, cochas y caños (Figura 1).

La población que habita en la Zona de Influencia del ACR AA en su mayoría pertenecen a los pueblos indígenas Yagua (30\%), Bora (29\%), Huitoto (25\%) y Ocaina $(5 \%)$, además de población mestiza $(11 \%)$ proveniente principalmente de Loreto y San Martín (Gore Loreto-DECDB, en prensa).

Desde el punto de vista cultural, los pueblos Huitoto, Bora y Ocaina, comparten rasgos culturales muy similares, debido a que forman parte de una misma familia lingüística, con un mismo origen e historia, manteniendo su identidad y compartiendo un patrón de organización social común, así como conocimientos y prácticas ancestrales de relación con la naturaleza propios de los pueblos indígenas del bosque tropical amazónico (Gore Loreto DECDB, en prensa). 


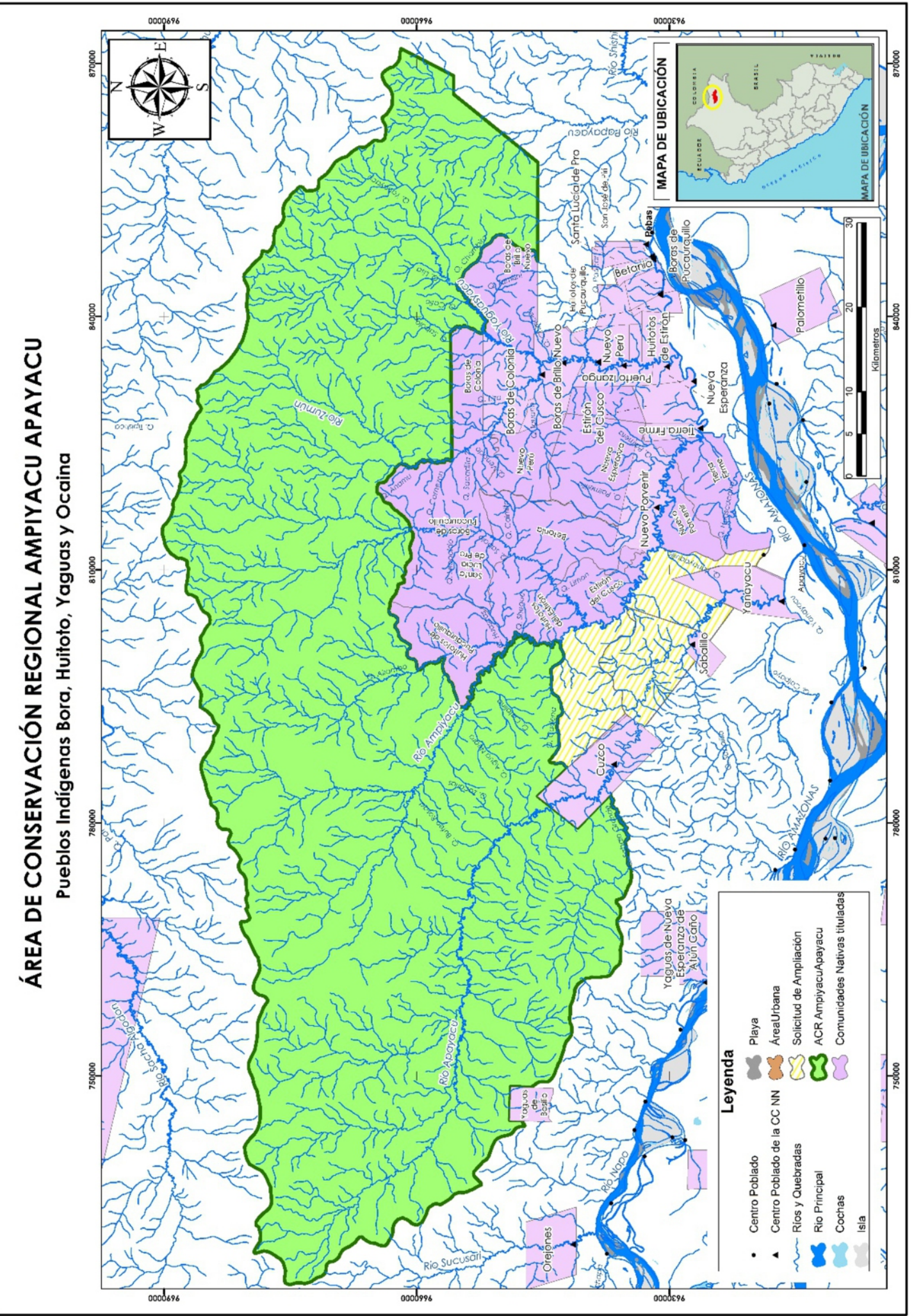

Figura 1. Representación del Área de Conservación Regional Ampiyacu Apayacu (verde) y su zona de influencia o de territorios comunales (morado). Fuente: Mapa elaborado por el Laboratorio de SIG del IBC, 2016. 
Las comunidades de los pueblos originarios Yagua, Bora, Huitoto y Ocaina están organizados a través de la Federación de Comunidades Nativas del Ampiyacu (FECONA).

Entre las principales actividades económicas y de subsistencia que practican las comunidades del Ampiyacu se encuentran la agricultura, la crianza de animales menores, la actividad forestal, el uso de recursos forestales no maderables, uso de plantas medicinales, turismo, venta de artesanías, pesca y caza de fauna silvestre (Gore Loreto - DECDB, en prensa).

El área donde se realizaron los censos de fauna silvestre con énfasis en los animales de caza se encontraron en la zona de aprovechamiento directo del ACRAA, por el curso superior del río Yaguasyacu. Los bosques se encuentran en proceso de recuperación luego de la excesiva presencia de extractores foráneos como habilitadores, madereros, cazadores, hasta antes del 2010 en que el ACR AA fue declarada como área natural protegida de carácter regional (Gore Loreto - Procrel, 2013).

En similitud a lo descrito por Pitmann et al. (2004), el área de evaluación se caracterizó por presentar bosques de colinas de tierra firme con colinas semi ondulantes, ondulantes y disectadas por planicies inundables de afluentes del río Yaguasyacu. En cuanto a los bosques a la orilla del Yaguasyacu estuvieron en su mayoría representados por planicies inundables con secciones eventualmente inundadas o bosques de restingas.

El piso inferior del bosque estuvo compuesto por vegetación herbácea y arbustiva entre $0.5 \mathrm{~m}$ a $5 \mathrm{~m}$ de alto, y estuvo mayormente representado por especies como: Geonoma sp., Heliconia sp., Duroia hirsuta, Bactris sp., helechos herbáceos, Dieffenbachia Obliqua, cuya visibilidad hacia el interior del bosque osciló entre $5 \mathrm{~m}$, cuando la vegetación fue densamente poblada, hasta $35 \mathrm{~m}$ de distancia cuando el bosque fue colinoso y ligeramente poblada por vegetación herbácea y arbustiva. El estrato medio compuesto por palmeras entre 15 a $20 \mathrm{~m}$ de alto estuvo mayormente representado por Iriartea deltoidea, Attalea butyracea, Astrocaryum javarense, Mauritia flexuosa, Oenocarpus bataua, O. mapora, Phytelephas macrocarpa, Astrocaryum chambira y Euterpe precatoria. El estrato superior entre $21 \mathrm{~m}$ a $30 \mathrm{~m}$ de alto, estuvo representado principalmente por especies leñosas como Matisia ochrocalyx, Simarouba amara, Carapa guianensis, Triplaris sp., Inga sp., Ormosia sp., Spondias mombin, Acacia sp., Aspidosperma exelsum, Virola sp., Cedrela odorata, Ocotea sp., Calycophyllum spruceanum e Hymenaea oblongifolia. Mientras que, el estrato emergente compuesto por árboles que superaron los $35 \mathrm{~m}$ de alto, estuvieron representados principalmente por Chorisia integrifolia, Apuleia leiocarpa, Calophyllum mariae, Hymenaea oblongifolia, Pachira aquatica, Maquira coriacea, Eschweilera sp., Cedrelinga catenaeformis y Cariniana domestica.

La identificación de las especies por la toponimia vernacular fue con la ayuda de guías locales capacitados y experimentados y la misma fue cotejada con la guía de plantas reportadas por el Field Museum de Chicago (Pitmann et al., 2004, 2011), y la guía de plantas de importancia económica y ecológica (Valderrama 2003).

\section{METODOLOGÍA EMPLEADA}

En este reporte se presentan las metodologías de monitoreo de fauna silvestre empleadas con participación de 14 comunidades situadas en la zona de influencia del ACR AA, específicamente en la cuenca del Ampiyacu y su afluente el Yaguasyacu.

Entre las herramientas de monitoreo empleadas se tuvo las siguientes:

\section{Registros de caza}

Esta metodología fue aplicada durante todo el 2016, con el propósito de estimar la abundancia y extracción de los animales cazados en el interior del ACR AA. El registro de caza consistió en anotar el nombre del cazador, especie cazada, sexo, número de animales cazados, fecha de salida a la caza, fecha de retorno de la caza y el sitio de caza. También se incluyó la edad del animal cazado en las categorías: cría, juvenil y adulto.

El registro de caza permitió recopilar, sistematizar y analizar cuantitativamente el nivel de uso preferente y los sitios de caza, con ese fin se capacitaron a comuneros de ambos sexos, estudiantes y profesores, en el llenado de las fichas de registro.

La información consignada en los registros de caza, fue analizada mediante la captura por unidad de esfuerzo (CPUE). El método de CPUE se usó para indicar la abundancia de los animales y si están siendo sobre cazados o no (Puertas \& Bodmer, 2004; Vickers, 1991). La CPUE examinó las relaciones entre esfuerzo y rendimiento. En este caso, la relación fue presentada como días de caza empleada por el cazador para conseguir un animal (Puertas \& Bodmer, 2004; Fang et al., 2008).

Cabe indicar que, mediante el registro de caza, se involucró a 17 cazadores en la colección de datos. Este método participativo ayudó al personal técnico y cazadores locales a encontrar caminos comunes para discutir asuntos tendientes a un mejor manejo de la fauna silvestre. 


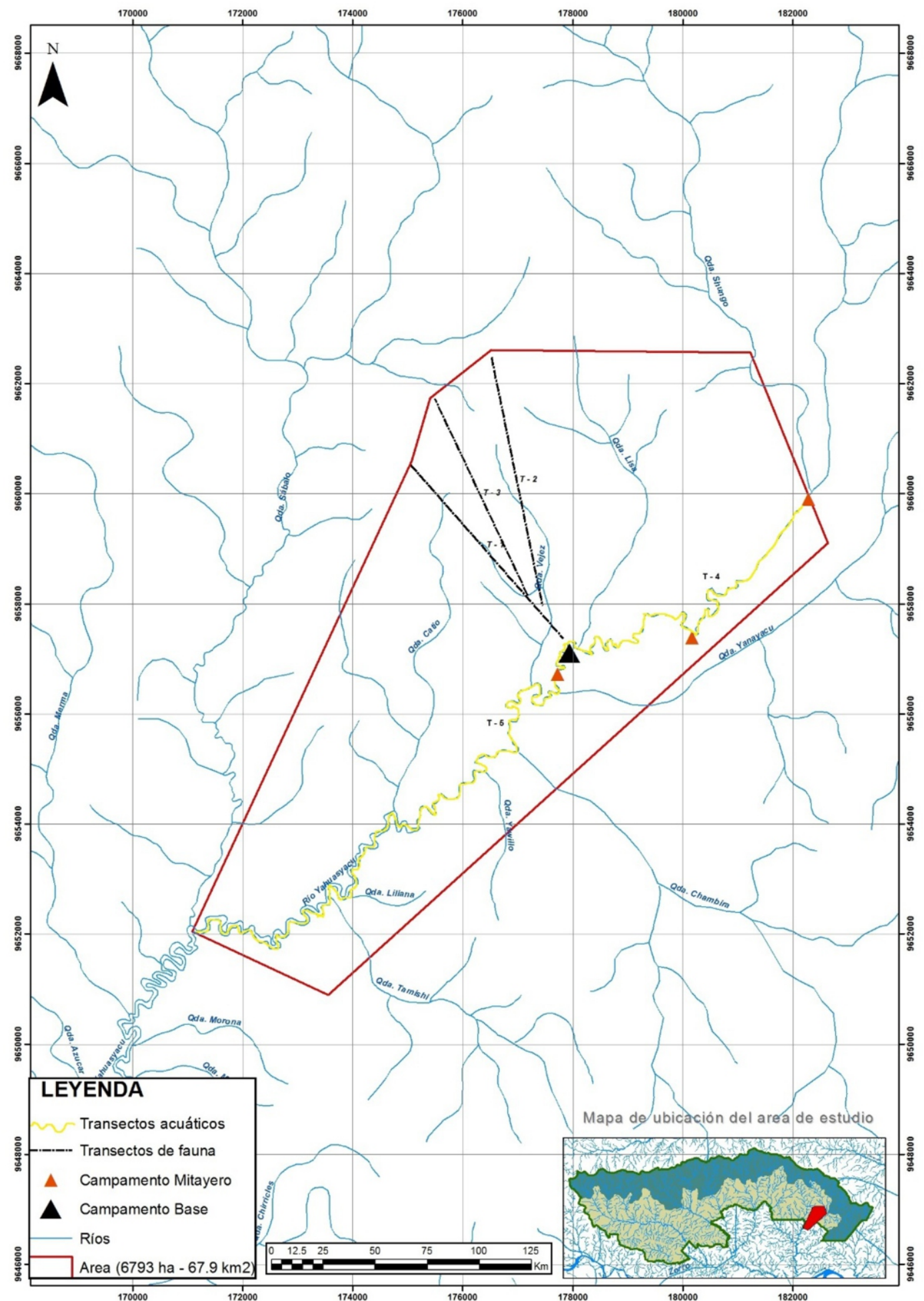

Figura 2. Localización del área de evaluación de los censos de animales de caza en el Yaguasyacu, interior del ACR Ampiyacu Apayacu. T1 = Transecto o Trocha 1, T2 = Transecto o Trocha 2 y T3 = Transecto 0 Trocha 3 (área muestreada $=5.64 \mathrm{Km}^{2}$ ). Lo resaltado en rojo representa el área de influencia en la zona de aprovechamiento directo $=67.9 \mathrm{Km}^{2}$. En amarillo se consigna los recorridos acuáticos como transectos acuáticos. T4 = Transecto acuático aguas arriba y T5 = Transecto acuático aguas abajo. 


\section{Diálogos interactivos}

Mediante los diálogos interactivos se posibilitó el intercambio de información con los comuneros sobre la aplicación de las herramientas metodológicas del monitoreo de fauna silvestre. Esto, con el fin de ayudar a identificar a los informantes o cazadores clave de la comunidad y de indagar la realización de actividades cotidianas, con respecto al uso de la fauna silvestre en chacras, bosques secundarios, aledaños y en el interior del área protegida.

Asimismo, para recibir información sobre las estrategias usadas en la cacería, herramientas de caza, sistema de comercialización y el papel de género en la cacería. En general, esta metodología a modo informal permitió percibir cualitativamente lo que piensa la gente de la actividad de caza que se viene realizando, el contexto político y legal de la caza y que oportunidades tendrían para continuar usando sosteniblemente los animales de caza.

La metodología se aplicó de forma espontánea durante las faenas cotidianas como trabajos comunales "mingas", comidas, visitas a la casa, encuentros casuísticos en la comunidad o en los mercados de Pebas e Iquitos, reuniones comunales y días festivos, entre otros eventos.

\section{Censos de fauna silvestre por transecto lineal}

La evaluación de los animales de caza se realizó mediante la conducción de censos terrestres y acuáticos. Los censos terrestres se realizaron por trochas o transectos que fueron lineales, mientras que los censos acuáticos o transectos acuáticos se realizaron aguas arriba y aguas abajo a lo largo del río Yaguasyacu a partir del campamento base (Figura 2).

Tres trochas fueron utilizadas durante las actividades censales con longitudes entre 4.5 a $5 \mathrm{~km}$ cada una. Las trochas fueron abiertas con el apoyo de guías de campo. Paralelamente, se colocaron cintas plásticas de colores convencionales cada 100 metros a fin de estimar la distancia recorrida y de facilitar la localización de los animales en la trocha.

Los censos acuáticos, fueron realizados en una embarcación de madera de $5 \mathrm{~m}$ de largo. Los censos fueron realizados con la participación de un profesional y dos asistentes de campo.

La actividad censal tanto acuática como terrestre fueron realizadas desde las 7:00 hasta las 8:00h y desde las 16:00 hasta 17:00h dependiendo de la longitud de los transectos y las condiciones climáticas.

Los censos fueron repetidos, ya que se consideró como nuevo censo el retorno por el mismo transecto, luego de un descanso de una hora como dando tiempo a que nuevas especies fueran observadas y se minimice cierto grado de perturbación. Durante los censos terrestres y acuáticos fueron recorridos un total de $322 \mathrm{~km}$ lineales, de los cuales $186 \mathrm{~km}$ fueron censos terrestres y $136 \mathrm{~km}$ fueron censos acuáticos.

Los censos acuáticos fueron conducidos también para complementar la presencia de animales con énfasis de hábitos arborícolas en bosques inundables en similitud a lo realizado en la Amazonia Brasilera (Haugaasen \& Péres 2005; Laufer et al., 2012), Los datos generales consignados al inicio del censo consistió en la siguiente información: fecha, día, lugar, número del transecto, nombre del cuerpo de agua y lugar de la unidad de muestreo, tipo de censo, condición atmosférica y hora de inicio y final del censo. Cuando un individuo fue detectado, se anotó la hora, especie, número de individuos o tamaño de grupo, distancia perpendicular del primer animal avistado a la trocha o a la orilla del río si el censo fue acuático, ubicación del animal avistado en la trocha, y tipo de hábitat. $\mathrm{Al}$ término del censo se anotó el total de la distancia recorrida.

Para la identificación de especies y estimaciones de biomasa se usó la información reportada para bosques inundables y de altura por Bodmer (1994), Bodmer et al. (1997, 2014), Aquino et al. (2001, 2013) y Aquino et al. (2015).

Especies de caza de hábitos nocturnos, cuyas densidades fueron limitadas de estimar mediante censos por transectos, éstas fueron estimadas mediante el método cartográfico o de localización en el mapa (Tellería, 1986). Este método consistió en registrar todos los avistamientos de huellas efectuados durante el recorrido simultáneo de tres trochas en un área de muestreo de $5.64 \mathrm{Km}^{2}$ a fin de estimar su abundancia por especie. La misma, conformada por la triangulación de tres trochas equidistantes entre sí. Para la diferenciación de un mismo ejemplar, las huellas fueron analizadas en razón a su localización en la trocha, hora de registro y dirección de desplazamiento. Este método fue utilizado para estimar la densidad poblacional de Tapirus terrestris, Mymecophaga tridactyla y Priodontes maximus. Ello, en vista que no se obtuvieron avistamientos directos de esas especies por sus hábitos nocturnos.

La densidad poblacional de Cuniculus paca fue estimada utilizando el método de conteo de madrigueras referida por Beck - King et al. (1999). El mismo, que consistió en contar el número de madrigueras durante los recorridos censales en un ancho fijo de $5 \mathrm{~m}$ y usando una constante de 3.5 que es el promedio de madrigueras que utiliza la especie en un kilómetro cuadrado. 


\section{ANÁLISIS DE DATOS}

\section{Densidadpoblacional}

Dado a que la mayoría de registros de especies tuvieron un rango inferior a 20 avistamientos, reportamos densidades usando el análisis de ancho fijo descrita por Bodmer et al. (2014). Eventualmente, cuando el tamaño de muestra fue superior a los 20 avistamientos, la densidad fue calculada usando el programa Distance, que está basada en el estimado de las distancias perpendiculares de detección (Buckland et al., 2004), cuya técnica es una de las más apropiadas para el caso de animales de caza en bosques tropicales y que según Peres (1999) también puede aplicarse con tamaño de muestras igual o mayor a 20 avistamientos; aunque las estimaciones actuales usando Distance sugieren tener como mínimo 60 avistamientos (Laake et al., 2006; Buckland et al., 2004).

En el caso de C. paca para la estimación poblacional se usó la adaptación del ancho fijo que es: $\mathrm{D}=(\mathrm{n} / 3.5) /(\mathrm{LW} 2)$, donde $\mathrm{n}$ es el número de madrigueras, L es la longitud del transecto y $\mathrm{W}$ es el ancho de búsqueda y “2” es la constante que indica la búsqueda a ambos lados (Beck- King et al., 1999; Pérez et al., 2016).

\section{Evaluación de la sostenibilidad}

Para el análisis de sostenibilidad de caza se hizo uso del modelo de cosecha unificado (Bodmer et al., 1997). Para este propósito, además de la presión de caza fue necesario determinar la productividad anual estimada y extrapolada de la literatura disponible para el nor oriente peruano (Bodmer et al., 1997; Fang et al., 2008), la cual fue calculada multiplicando la productividad bruta (número de embriones y/o fetos/hembras adultas analizadas) por el número de de gestaciones al año y por la media de la densidad poblacional (Aquino et al., 2001; Hurtado-Gonzáles \& Bodmer, 2004; Mayor et al., 2007, 2009, 2011; Rengifo et al., 1996).

\section{RESULTADOS Y DISCUSIÓN}

Con el propósito de levantar información para el establecimiento de una línea base con fines de implementar las actividades de monitoreo y establecer planes de manejo de fauna silvestre orientado a los animales de caza es que se realizó el presente estudio.

\section{ANIMALES EXTRAÍDOS Y ABUNDANCIA}

Durante el 2016, un total de 51 especies de animales fueron utilizadas en la alimentación y venta como carne de monte por 14 comunidades de la FECONA. Los animales cazados totalizaron 2584 ejemplares que hicieron una biomasa bruta de $37,992.6 \mathrm{~kg}$. Entre las especies que presentaron mayor índice de abundancia y resultaron más fáciles de cazar destacan: C. paca, T. pecari, D. fuliginosa y P. tajacu (Figura 3). Es decir, indica una abundancia de animales preferidos de caza y que estarían resultando más fáciles de cazar. Cabe indicar que, hoy en día las comunidades están realizando el control y vigilancia limitando el acceso a infractores, lo que conlleva a que disminuya la caza al interior del ACR.

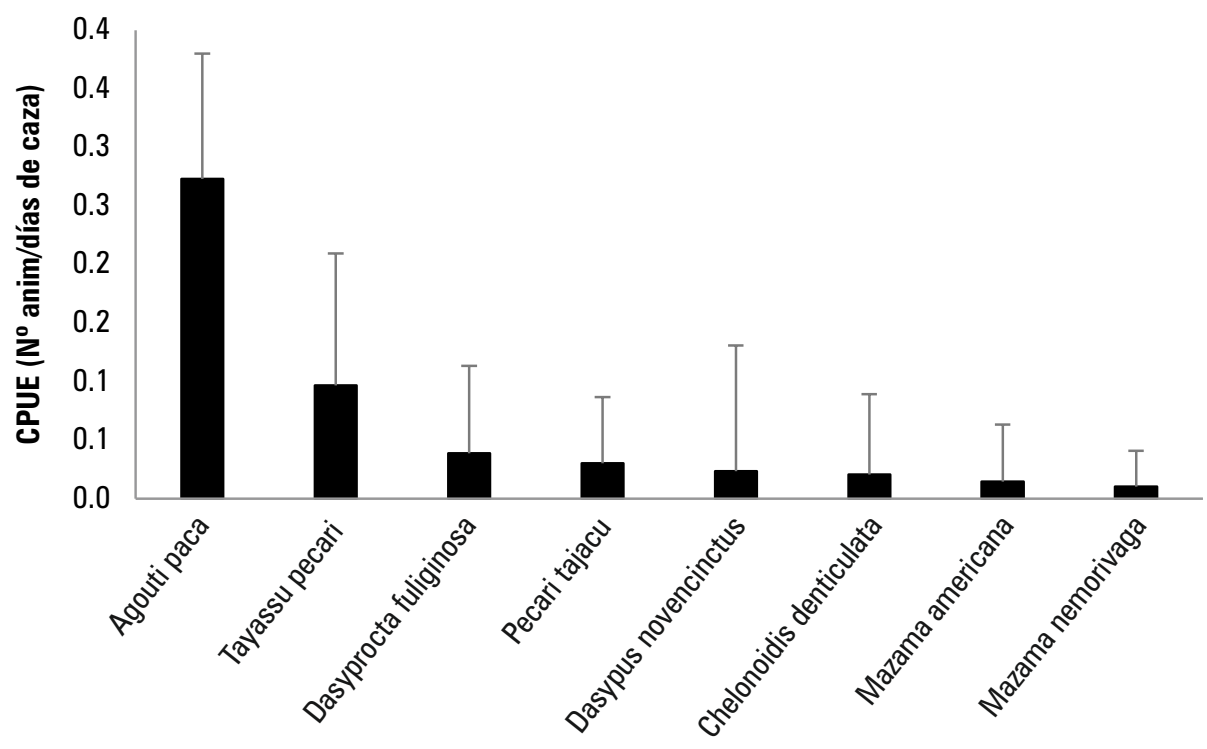

Figura 3. Captura por Unidad de Esfuerzo (CPUE) de las especies más abundantes y fáciles de cazar por cazadores de 14 comunidades de FECONA en el ACRAA durante el 2016. 
Tabla 1. Densidad y biomasa de mamíferos y aves de importancia económica y de subsistencia en el Yaguasyacu, zona de aprovechamiento directo del ACRAA.

\begin{tabular}{|c|c|c|c|c|c|c|}
\hline $\mathbf{N}^{0}$ & Especies & Nombre común & Peso & $\begin{array}{c}\text { Tamaño } \\
\text { de grupo }\end{array}$ & $\begin{array}{c}\text { Densidad } \\
(\text { Ind-//km²) }\end{array}$ & $\begin{array}{c}\text { Biomasa } \\
\text { poblacional } \\
\left(\mathrm{kg} / \mathrm{km}^{2}\right)\end{array}$ \\
\hline & Ungulados & & & & & \\
\hline 1 & Tayassu pecari & Huangana & 33 & 9 & $3.2^{1}$ & 105.6 \\
\hline 2 & Pecari tajacu & Sajino & 25 & 2.5 & $1.3^{2}$ & 32.5 \\
\hline 3 & Mazama americana & Venado colorado & 33 & 1 & 0.711 & 23.43 \\
\hline 4 & Mazama nemorivaga & Venado cenizo & 20 & 1 & $0.34^{2}$ & 6.7 \\
\hline \multirow[t]{3}{*}{5} & Tapirus terrestris & Tapir o sachavaca & 160 & 1 & $0.53^{1}$ & 84.8 \\
\hline & Sub-total & & & & & 253.03 \\
\hline & Primates & & & & & \\
\hline 6 & Leontocebus nigricollis & Pichico negro & 0.6 & 6.2 & $4.81^{3}$ & 2.89 \\
\hline 7 & Saimiri macrodon & Fraile & 0.8 & 25 & $10.93^{2}$ & 8.74 \\
\hline 8 & Cebus yuracus & Mono blanco & 3 & 8.5 & $2.34^{2}$ & 7.02 \\
\hline 9 & Cheracebus lucifer & Tocón negro & 1.5 & 3.6 & $2.47^{2}$ & 3.7 \\
\hline 10 & Pithecia hirsuta & Huapo negro & 2 & 2.8 & $1.32^{2}$ & 2.64 \\
\hline 11 & Alouatta seniculus & Mono coto & 8 & 7 & $3.15^{2}$ & 25.16 \\
\hline \multirow[t]{3}{*}{12} & Lagothrix lagothricha & Mono choro & 11 & 12.6 & $10.28^{2}$ & 113.08 \\
\hline & Sub-total & & & & & 163.23 \\
\hline & Roedores & & & & & \\
\hline 13 & Dasyprocta fuliginosa & Añuje & 3 & 1.2 & $2.2^{2}$ & 6.59 \\
\hline 14 & Myoprocta pratti & Punchana & 2 & 1 & $0.68^{2}$ & 1.35 \\
\hline \multirow[t]{3}{*}{15} & Agouti paca & Majás & 9 & 1 & $4.2^{4}$ & 37.8 \\
\hline & Sub-total & & & & & 45.74 \\
\hline & Edentados & & & & & \\
\hline 16 & Myrmecophaga tridactyla & Oso hormiguero & 31 & 1 & $0.35^{1}$ & 10.85 \\
\hline 17 & Bradypus variegatus & Perezoso de tres dedos & 4.5 & 1 & $2.04^{2}$ & 9.16 \\
\hline \multirow[t]{3}{*}{18} & Priodontes maximus & Yangunturo, carachupa mar & anAABA & 1 & $0.18^{1}$ & 7.99 \\
\hline & Sub-total & & & & & 28 \\
\hline & Aves de caza & & & & & \\
\hline 19 & Harpia harpyja & Águila harpía & 4.8 & 1 & $0.13^{2}$ & 0.62 \\
\hline 20 & Psophia crepitans & Trompetero & 1 & 6 & $8.07^{2}$ & 8.07 \\
\hline 21 & Pipile cumanensis & Pava del monte & 1.3 & 1.5 & $0.93^{2}$ & 1.21 \\
\hline 22 & Penelope jacquacu & Pucacunga & 1.3 & 2 & $2.42^{2}$ & 3.15 \\
\hline 23 & Nothocrax urumutum & Montete & 1.25 & 1 & $0.13^{2}$ & 0.16 \\
\hline 24 & Mitu salvini & Paujil & 3 & 2 & $0.45^{2}$ & 1.35 \\
\hline 25 & Crypturellus undulatus & Panguana & 0.5 & 1 & $1.75^{2}$ & 0.88 \\
\hline \multirow[t]{3}{*}{26} & Tinamus major & Perdiz & 1 & 1 & $8.95^{3}$ & 8.9 \\
\hline & Sub-total & & & & & 24.34 \\
\hline & TOTAL & & & & & 514.34 \\
\hline
\end{tabular}

${ }^{1}$ Densidad estimada por el método cartográfico o de localización en el mapa.

${ }^{2}$ Densidad estimada de los métodos de transecto con anchura variable y anchura fija.

${ }^{3}$ Densidad estimada usando el programa Distance 5.0.

${ }^{4}$ Densidad obtenida mediante el conteo de madrigueras de Cuniculus paca "majás" 


\section{DENSIDAD POBLACIONAL YBIOMASA}

Un total de 25 especies de animales de caza entre ungulados (artiodáctilos), primates, roedores, edentados y aves de caza fueron registradas durante las actividades censales en el río Yaguasyacu, interior del ACR AA (Tabla 1). Las especies más representadas fueron: Saimiri macrodon (10.93 ind $\left./ \mathrm{km}^{2}\right)$, Lagothrix lagothricha $\left(10.28 \mathrm{ind} / \mathrm{km}^{2}\right)$, Tinamus major (8.95 ind $\left./ \mathrm{km}^{2}\right)$, Psophia crepitans (8.07 ind $\left./ \mathrm{km}^{2}\right)$ y Leontocebus nigricollis (4.81 ind $/ \mathrm{km}^{2}$ ), (Tabla 1 ).

Los ungulados, especies de gran importancia económica, tuvieron cinco especies representadas. Tayassu pecari (3.2 ind $/ \mathrm{km}^{2}$ ) fue la especie que presentó mayor densidad poblacional, seguido de Pecari tajacu $\left(1.3 \mathrm{ind} / \mathrm{km}^{2}\right)$, Mazama americana $\left(0.71 \mathrm{ind} / \mathrm{km}^{2}\right)$, Tapirus terrestris $\left(0.53 \mathrm{ind} / \mathrm{km}^{2}\right)$ y M. nemorivaga $\left(0.34 \mathrm{ind} / \mathrm{km}^{2}\right)$.

Respecto a la biomasa poblacional, el total calculado fue de $476.54 \mathrm{~kg} / \mathrm{km}^{2}$ (Tabla 1). De ese total a nivel de especies, $113.08 \mathrm{~kg} / \mathrm{km}^{2}$ (24\%) correspondieron a L. lagothricha, seguido de $T$. pecari con $105.6 \mathrm{~kg} / \mathrm{km}^{2}(22 \%)$, T. terrestris con $84.8 \mathrm{~kg} / \mathrm{km}^{2}$ (18\%), P. tajacu con $32.5 \mathrm{~kg} / \mathrm{km}^{2}(7 \%)$, A. seniculus con $25.16 \mathrm{~kg} / \mathrm{km}^{2}$ y M. americana con $23.43 \mathrm{~kg} / \mathrm{km}^{2}(5 \%$, respectivamente), (Tabla 1$)$.

A nivel de grupo taxonómico, en la Tabla 1 se puede apreciar que los ungulados fueron los más representados en términos de biomasa con 253.03 $\mathrm{kg} / \mathrm{km}^{2}$ (55\%), seguido de los primates con 162.9 $(35 \%)$.

Los registros censales obtenidos en el río Yaguasyacu, en el interior del ACR Ampiyacu Apayacu de acuerdo a la zonificación establecida en el plan maestro de dicha área de conservación correspondieron a la zona de aprovechamiento directo (Gorel/Procrel, 2013). Es en esa área donde se viene realizando el aprovechamiento directo de recursos naturales realizado por la población local de la zona de influencia del ACRAA mediante la aplicación de los lineamientos de uso de recursos naturales, excluyendo la caza de aquellas especies objeto de conservación como: $P$. maximus "yangunturo", T. terrestris "sachavaca", Myrmecophaga tridactyla "oso hormiguero", $L$. lagothricha "mono choro" y Harpia harpyja "águila harpía".

Los resultados de densidades reportados en este estudio comparado con lo reportado por Aquino et al. (2007), para el río Algodón, cuenca media del Putumayo muestran una mayor abundancia en términos de densidad de animales de caza de importancia económica y de subsistencia en el algodón que en el Yaguasyacu, aunque al parecer, el sitio del río Algodón - Putumayo por su ubicación geográfica más distante a centros poblados, estuvo sujeta a menor presión de caza que la zona evaluada en el Yaguasyacu que si está teniendo mayor concurrencia de caza. Entre los ungulados, las densidades fueron inferiores en P. tajacu y T. pecari, similares en $M$. americana y ligeramente inferior a lo registrado para T. terrestris. En cuanto a primates todos los registros de densidades fueron inferiores a lo reportado para el río Algodón, a excepción de Cheracebus lucifer "tocón negro" que fue superior en el Yaguasyacu. En cuanto a los roedores, las densidades obtenidas en el Yaguasyacu referidas a D. fuliginosa fueron superiores en el Yaguasyacu que en el Algodón, caso contrario ocurrió con Myoprocta pratti. Respecto a las aves de caza, las densidades de Mitu salvini, Penelope jacquacu y Pipile cumanensis fueron superiores en el algodón, sin embargo las densidades de P. crepitans y T. major fueron superiores en el Yaguasyacu. Cabe indicar que en el Yaguasyacu se registró la presencia de $H$. harpyja "águila harpía", especie emblemática que es considerado como objeto de conservación del ACRAA, cuyos registros en términos de densidad aún no fueron reportados para el nor oriente peruano.

Comparando las densidades obtenidas en este reporte con lo registrado por Pérez (2012) para la zona de aprovechamiento directo de los ríos Tahuayo - Blanco, en el Área de Conservación Regional Comunal Tamshiyacu Tahuayo (ACRCTT), se tuvo en cuanto a ungulados los mayores registros de T. pecari, M. americana y $T$. terrestris, con registro similar en venado gris e inferior en $P$. tajacu. A nivel de primates, aunque ciertas especies son diferentes entre ambos sitios, los registros de densidad fueron superiores en el ACRCTT que en el Yaguasyacu, a excepción de $L$. lagothricha, cuya densidad poblacional duplicó a lo reportado para la zona de aprochamiento del ACRCTT. Cabe indicar que el ACRCTT posee una de las mayores diversidades de primates reportadas para áreas de conservación en el Peru (Puertas \& Bodmer, 1993). La dominancia de S. macrodon de tamaño pequeño envés de especies de primates de tamaño grande indicaría que estas especies aún estarían en proceso de recuperación poblacional luego de la fuerte presión de caza que estuvieron sujetas antes del establecimiento como ACRAA (Gore Loreto - PROCREL, 2013), en contraste a otras áreas como la Zona de aprovechamiento directo de la Reserva Nacional Pucacuro, donde las densidades de L. lagothricha son mayores (Pérez et al., 2016). Diferencias de densidades de primates entre una y otra área también pueden deberse a los tipos de hábitats con mayor producción de frutos y que necesitan ser evaluados. En cuanto a los roedores $D$. fuliginosa y $M$. pratti fueron superiores 
en el Yaguasyacu que en el ACRCTT. Respecto al edentado $M$. tridactyla, el registro de densidad fue similar a la reportado para la zona fuente del ACRCTT ya que no tuvo registro para la zona de aprovechamiento directo. En cuanto a las aves de caza los mayores registros fueron para el Yaguasyacu que para el ACRCTT. Es decir, con mayores registros de densidad de $P$. crepitans, $P$. cumanensis y $T$. major; con similar densidad de $M$. salvini, e inferior densidad en P. jacquacupu.

\section{SOSTENIBILIDAD DE LA CAZA}

En la zona de aprovechamiento directo, fueron seis las especies más preferidas por la población local que soportan la presión de caza y se encuentran por debajo del límite de cosecha sostenible. Estas especies son: P. tajacu, T. pecari, M. americana, $M$. nemorivaga, $C$. paca y D. fuliginosa (Tabla 2). Es decir, son las especies que pueden ser aprovechadas con planes de manejo facultados por la autoridad competente.

El análisis de sostenibilidad de los animales de caza fue realizado con el modelo de cosecha unificado (Bodmer, 2001) en el interior del ACR AA y se efectuó en base a las seis especies más preferidas de caza, sobre un área estimada de $544.9 \mathrm{~km}^{2}$ situada en la zona de aprovechamiento directo y comprendido en los cursos superiores de los ríos Yaguasyacu y Zumún, que se encuentran limitando el río Ampiyacu propiamente. En dicha área, los animales cazados tuvieron un rango de 0.34 a 4.2 ind $/ \mathrm{km}^{2}$ (Tabla 2).

Según el análisis de sostenibilidad de los animales de caza, se tuvo que la producción cosechada de las seis especies preferidas de caza se encontró por debajo del límite de cosecha máxima sostenible-MSY (Tabla 2).

Los resultados obtenidos del análisis de sostenibilidad en este estudio permitieron recomendar la aplicación de cuotas de aprovechamiento temporal de animales de caza procedentes del ACR AA por un período de seis meses (abril a setiembre, 2017) especificadas en un informe técnico sustentatorio hasta que se elabore e implemente un Plan de Manejo de Fauna Silvestre (Resolución Gerencial Regional-001-2017-GRLGGR-ARA LORETO). Esta aplicación incluyó a las 14 comunidades de FECONA participantes en las actividades de monitoreo de fauna silvestre. Como medida precautoria, las cuotas de aprovechamiento de uso de las seis especies que soportan la presión de caza como P. tajacu, T. pecari, M. americana, $M$.

Tabla 2. Sostenibilidad de la caza dentro del ACR Ampiyacu Apayacu, zona de aprovechamiento directo.

\begin{tabular}{|c|c|c|c|c|c|c|}
\hline \multirow[b]{2}{*}{ Parámetros } & \multicolumn{6}{|c|}{ ESPECIES } \\
\hline & $\begin{array}{l}\text { Pecari } \\
\text { tajacu }\end{array}$ & $\begin{array}{c}\text { Tayassu } \\
\text { pecari }\end{array}$ & $\begin{array}{c}\text { Mazama } \\
\text { americana }\end{array}$ & $\begin{array}{c}\text { Mazama } \\
\text { nemorivaga }\end{array}$ & $\begin{array}{c}\text { Cuniculus } \\
\text { paca }\end{array}$ & $\begin{array}{c}\text { Dasyprocta } \\
\text { fuliginosa }\end{array}$ \\
\hline $\begin{array}{l}\text { Área de caza en la zona de } \\
\text { aprovechamiento directo }(\mathrm{km})\end{array}$ & $544.9 \mathrm{~km}^{2}$ & $544.9 \mathrm{~km}^{2}$ & $544.9 \mathrm{~km}^{2}$ & $544.9 \mathrm{~km}^{2}$ & $544.9 \mathrm{~km}^{2}$ & $544.9 \mathrm{~km}^{2}$ \\
\hline Animales cazados $x$ año & 49 & 55 & 30 & 8 & 511 & 1 \\
\hline Densidad $\left(\mathrm{km}^{2}\right)$ & 3.2 & 1.3 & 0.71 & 0.34 & 4.2 & 2.2 \\
\hline $1 / 2$ densidad ind $/ \mathrm{km}^{2}$ & 1.6 & 0.65 & 0.36 & 0.17 & 2.1 & 1.1 \\
\hline$N^{\circ}$ de gestaciones/año & $1.69^{5}$ & $1.88^{5}$ & $1.7^{4}$ & $1.7^{4}$ & $2^{6}$ & $2^{5}$ \\
\hline $\begin{array}{l}\text { Productividad Total (crías } x \\
\text { hembras) o la Productividad } \\
\text { Bruta (PB) }\end{array}$ & $0.76^{1}$ & $0.57^{2}$ & $0.48^{3}$ & $0.54^{4}$ & $0.79^{6}$ & $1^{5}$ \\
\hline Producción anual (PA) & 2.055 & 0.69654 & 0.29376 & 0.15606 & 3.318 & 2.2 \\
\hline Presión de caza (ind/km2) & 0.0899 & 0.1009 & 0.0551 & 0.01468 & 0.93779 & 0.0018 \\
\hline Producción cosechada (\%) & 4.4 & 14 & 19 & 9 & 28 & 0.1 \\
\hline $\begin{array}{l}\text { Límite de la cosecha } \\
\text { sostenible MSY \% }\end{array}$ & 40 & 40 & 40 & 40 & 60 & 60 \\
\hline Sostenibilidad & $\mathrm{Si}$ & $\mathrm{Si}$ & $\mathrm{Si}$ & $\mathrm{Si}$ & $\mathrm{Si}$ & $\mathrm{Si}$ \\
\hline
\end{tabular}

'Mayor et al. (2007), ' ${ }^{2}$ Mayor et al. (2009), , ${ }^{3}$ Mayor et al. (2011), 'Hurtado-Gonzales \& Bodmer (2004),

${ }^{5}$ Aquino et al. (2001), ${ }^{6}$ Rengifo et al. (1996). 
nemorivaga, C. paca y D. fuliginosa, se consideró a lo estipulado en los "Lineamientos de uso de recursos naturales en el Área de Conservación Regional Ampiyacu Apayacu", aprobado mediante Resolución Directoral $\mathrm{N}^{\circ}$ 021-2013-GGRPOCREL/DE, de fecha 07 de noviembre del 2013. Una primera experencia de manejo de fauna silvestre orientada a la comercialización de carne de monte de aquellas especies que soportan la presión de caza está existosamente siendo implementada en la Reserva Nacional de Pucacuro (Pérez et al. 2016), que es un área natural protegida cuya administración nacional depende del Servicio Nacional de Áreas Naturales Protegidas por el Estado (SERNANP); sin embargo, la experiencia piloto que está siendo implementada en el ACRAA es la única dada para las áreas de conservación regional en la amazonía de Loreto, cuya administración depende de la Autoridad Regional Ambiental de Loreto (ARA Loreto) de cuyas lecciones aprendidas indudablemente serán aplicadas con las adaptaciones del caso a otras áreas de conservación regional.

La sostenibilidad de las especies fueron analizadas para determinar, si éstas estuvieron siendo cazadas de forma sostenible o no. En nuestro caso utilizamos el modelo de sostenibilidad de cosecha unificado, con el propósito de evaluar la sostenibilidad de la caza actual y el potencial para el uso sostenible a largo plazo, combinando el modelo de reclutamiento del stock y de cosecha (Fang et al., 2008). Futuras actividades de monitoreo a ser realizadas, posibilitarán el entendimiento en el nivel de aprovechamiento y las tendencias poblacionales de las especies preferidas de caza y que posibilitarán su discusión con otras áreas de la Amazonía peruana.

\section{CONCLUSIÓN}

- Un total de 52 registradores de caza reportaron la cacería practicada por 152 cazadores pertenecientes a 14 comunidades de la cuenca del Ampiyacu, incluyendo su afluente el Yaguasyacu durante el 2016. Entre los resultados destacan el registro de 2584 animales cazados que representan una biomasa bruta extraída de $37,992.6 \mathrm{~kg}$. Es decir, los bosques del ACR AA poseen una alta producción de fauna silvestre, representando en consecuencia un importante servicio ecosistémico que está contribuyendo a la seguridad alimentaria de la población local.

- Según la Captura por Unidad de Esfuerzo se tuvo que C. paca, T. pecari, D. fuliginosa, P. tajacu, $M$. americana, $M$. nemorivaga, L. lagothricha, D. novemcinctus, M. salvini, P. jacquacu $y$
Chelonoides denticulata fueron los animales más abundantes de caza.

- Un total de 25 especies de animales de caza entre ungulados (artiodáctilos), primates, roedores, edentados y aves de caza fueron registrados durante los censos de fauna silvestre en la zona de aprovechamiento directo del ACRAA.

- En la zona de aprovechamiento directo del ACR AA, seis especies fueron las preferidas por la población local que soportan la presión de caza y se encuentran por debajo del límite de cosecha sostenible. Estas especies son: P. tajacu, T. pecari, M. americana, M. nemorivaga, C. paca y D. fuliginosa; las mismas que pueden ser aprovechadas sosteniblemente con un plan de manejo de fauna silvestre.

\section{AGRADECIMIENTO}

Expresamos nuestro especial agradecimiento por sus valioso apoyo técnico y logístico a la Dirección Ejecutiva de Conservación de la Diversidad Biológica (DECDB/ Autoridad Regional Ambiental (ARA) del Gobierno Regional de Loreto en la persona del Lic. Alejandro Pinedo, al Instituto del Bien Común (IBC) en las personas de Ing. Ana Rosa Sáenz, Blgo. Genoveva Freitas, Blgo. Magaly del Risco, Ing. José David Urquiza y Sr. René Vásquez. Asimismo, agradecemos la participación activa del Presidente del Comité de Gestión del ACR, Sr. Rolando Panduro Mibeco; a los dirigentes y comunidades de FECONA, bajo la presidencia de la Sra. Liz Chicaje Churay; al Secretario de Manejo de Recursos Naturales y Territorio, Sr. José Luís Flores Robledo; a los profesores y alumnos de los centros educativos de las comunidades de la cuenca del Ampiyacu; a la estudiante Thalia Ruíz, quienes apoyaron como asistentes de campo; así como en la organización, logística y revisión del presente documento. Finalmente a la Fundación Gordon and Betty Moore por el financiamiento del presente estudio.

\section{BIBLIOGRAFÍA CITADA}

Aquino, R.; Cornejo, F.; Cortéz, L.; Encarnación, F.; Heymann, E.; Marsh, L.; Mittermeier, R.; Rylands, A.; Vermeer, R. 2015. Guía de Identificación de Bolsillode los Primates de Perú. Mittermeier, R.; Rylands, A. (Eds). Conservación Internacional, Series de Guías Tropicales de Bolsillo. Virgina, USA.22pp.

Aquino, R., Pezo, E.; Navarro, R.; Terrones, C.; Terrones, W. 2013. Análisis de la sostenibilidad de la caza en mamíferos mayores de la cuenca del 
río alto Itaya. En: Aquino, R.; Pezo, E.; y Gil, D.E. (Eds). Mamiferos de la cuenca del río alto Itaya, Amazonía peruana: Diversidad, Ecología, Demografiay Caza. p. 276-294.

Aquino, R.; Bodmer, R.E.; Gil, G. 2001. Mamíferos de la cuenca del río Samiria: ecología poblacional y sustentabilidad de la caza. Publicación Junglevagt for Amazonas, AIFWWF/DK - WCS. Lima, Perú. 108pp.

Beck-Kim, H.; von Helversen, O.; Beck-Kim, R. 1999. Home range, population density, and food resources of Agouti paca (Rodentia: Agoutidae) in Costa Rica: A study using alternative methods. Biotrópica 31 (4): 675-685.

Bodmer, R.; Fang, T.G.; Puertas, P.; Antúnez, M.; Chota, K.; Bodmer, W. 2014. Cambio Climático y Fauna Silvestre en la Amazonía Peruana: Impacto de la Sequía e Inundaciones Intensas en la Reserva Nacional Pacaya Samiria. FundAmazonia/Sernanp. FundAmazonia - Wust Ediciones.254pp.

Bodmer, R.E. 2001. Evaluating wildlife sustainable use in the Amazon: The unified harvest model. En: Sánchez, P.; Morales, A.; López, H.F. (Eds). Proceedings of the Fifth International Congress on Wildlife Management in Amazon and Latin America. Bogotá: Fundación Natura. 55pp.

Bodmer, R.; Aquino, R. 2000. Ecología poblacional de mamíferos en bosques inundables de la Amazonía Peruana. En: Cabrera, E.; Mercolli, C.; y Resquín, R (Eds). Manejo de Fauna Silvestre en Amazonía y Latinoamérica. Asunción, Paraguay. 578pp.

Bodmer, R.; Aquino, R.; Puertas, P. 1997. Alternativas de Manejo para la Reserva Nacional Pacaya - Samiria. Un Análisis sobre el Uso Sostenible de la Caza. En: Fang, T. Bodmer, R.; Aquino, R.; y Valqui, M. (Eds). Manejo de Fauna Silvestre en la Amazonía. La Paz, Bolivia. p. 6574.

Bodmer, R. 1994. Managing Wildlife with Local Communities in the Peruvian Amazon: The Case of the Reserva Comunal Tamshiyacu Tahuayo. En: Western, D.; Wright, R.M.; Strum, S. (Eds). Natural Connections: Perspectives in Community-based Conservation. Island Press. Washington D.C., Covelo, California, USA. p.113-134.

Bodmer, R., Fang, T.G.; Moya, L.; Gill, R. 1994. Managing Wildlife to Conserve Amazonian Forests: Population Biology and Economic Considerations of Game Hunting. Biological Conservation, 67:29-35.
Buckland, S.T.; Anderson, D.; Burnham, K.; Laake, J. 1993. Distance Sampling: Estimating the Abundance of Biological Populations. Chapman \& Hall, London. 441pp.

Burnham, K.; Anderson, D.; Laake, L. 1980. Estimation of density from line transect sampling of biological populations. Wildlife Monographs. $72 \mathrm{pp}$.

Fang, T.; Bodmer, R.; Puertas, P.; Mayor, P.; Pérez, P.; Acero, R.; Hayman, D. 2008. Certificación de pieles de pecaríes en la Amazonía peruana: Una estrategia para la conservación y manejo de fauna silvestre en la Amazonia peruana. Wust Ediciones. Lima, Perú. 203pp.

Gore Loreto-DECDB. En prensa. Plan Maestro del Área de Conservación Regional Ampiyacu Apayacu y su zona de influencia 2017 - 2021.

Gorel-Procrel, 2013. Plan Maestro del Área de Conservación Regional Ampiyacu - Apayacu 2012 - 2016. 125pp.

Haugaasen, T.; Peres, C. 2005. Primate assemblage structure: Amazonian Flooded and Unflooded Forests. American Journal of Primatology, 67: 243-258.

Hurtado, J.; Bodmer, R. 2004. Reproductive biology of female Amazonian brocket deer in northeastern Peru. European Journal of Wildlife Research, 52. 171-177.

Laake, J.F.; Buckland, S.T.; Anderson, D.R.; Burnham, K.P. 1994. Distance sampling: Abundance estimation of biological populations - Distance User's Guide. Colorado Cooperative Fish \& Wildlife Research. Unit, Colorado State University, Fort Collins, USA. 84pp.

Laufer, J.; Amador, J.A.; Conceicao, P.C.; Norris.; D.; Michalski, F. 2012. Short Communication: Use of boat surveys to provide complementary data on the ecology of Bradypus tridactylus (Pilosa: Bradypodidade) from northern Amazonia. Edentata, 13:56-60.

Mayor, P.; Pérez, P.; Bowler, M.; Puertas, P.; Kirkland, M.; Bodmer, R. 2015. Effect of selective logging on large mammals population in a remote indigenous territory in the northeastern Peruvian Amazon. Ecology and Society, 20 (4):36.

Mayor P.; Bodmer, R.E.; López-Béjar, M.; LópezPlana, C. 2011. Reproductive biology of the wild red brocket deer (Mazama americana) female in the Peruvian Amazon. Animal Reproduction Science, 128: 123-128.

Mayor P.; Bodmer, R.E.; Lopez-Béjar, M. 2009. Reproductive performance of the wild white- 
lipped peccary (Tayassu pecari) female in the Peruvian Amazon. European Journal of Wildlife Research, 55:631-634.

Mayor, P.; Guimarães, D.A.;Le Pendu, Y.; da Silva, J.V.; Jori, F.; López-Béjar, M. 2007. Reproductive performance of captive collared peccaries (Tayassu tajacu) in the eastern Amazon. Animal Reproduction Science, 102 (12): 88-97.

Pérez, P., Gonzáles, C.; Trigoso, M. 2016. Evaluación del plan de manejo de animales de caza en la Reserva Nacional Pucacuro. Folia Amazónica, 25 (1): 1-16.

Pérez, P. 2012. Funcionalidad del Área de Conservación Regional Comunal Tamshiyacu Tahuayo: Un análisis integral de la sostenibilidad de la caza. Reporte Técnico Yavacus, WCS. Iquitos, Perú. 46pp.

Pitman N.; Smith, R.C.; Vriesendorp, C.; Moskovits, D.; Piana, R.; Knell, G.; Watcher, T. 2011. Rapid Biological and Social Inventories Report 23. The Field Museum Chicago. 278 pp.

Pitman, N.; Smith, R.C.; Vriesendorp, C.; Moskovits, D.; Piana, R.; Knell, G.; Watcher, T. 2004. Rapid Biological Inventories: 12 - Perú: Ampiyacu, Apayacu, Yaguas, Medio Putumayo. The Field Museum of Chicago. 273 pp.

Puertas, P.E.; Bodmer, R. 2004. Hunting effort as a tool for community-based wildlife management in Amazonia. En: Silvius, K.; Bodmer, R.E.; Fragoso, J(Eds). Nature: wildlife conservation in South and Central America. Columbia University, New York, USA. p. 123-135.
Puertas, P.E.; Bodmer, R. 1993. Conservation of a high diversity primate assemblage. Biodiversity and Conservation, 2: 586-593.

Rengifo M. E.; Navarro, D.T.; Urrunaga, A.B. 1996. Crianza familiar del majás o paca (Agouti paca) en la Amazonía. Tratado de Cooperación Amazónica-Secretaría Pro Témpore No. 48. Mirigraf S.R.L., Lima, Perú. 43pp.

Robinson, J.; Bodmer, R. 1999. Towards wildlife management in tropical forests. Journal of Wildlife Management, 63: 1-13.

Tellería, J.L. 1986. Métodos de censo en vertebrados terrestres. Facultad de Biología, Universidad Complutense, Madrid-España. 32 pp.

Valderrama, H. 2003. Plantas de importancia económica y ecológica en el jardín botánico arboretum - Arboretum el Huayo, Iquitos, Perú. Folia Amazónica, 14 (1): 151-175.

Vickers, W. 1991. Hunting yields and game composition over ten years in an Amazonian village. En: Robinson, J.; Redford, K. (Eds). Neotropical Wildlife Use and Conservation. University of Chicago Press, Chicago, USA. p. 53-81.

Wilson, D.E.; Cole, F.; Nichols, J.; Rudran, R.; Foster, M.S. 1996. Measuring and monitoring biological diversity: Standard methods for mammals. Smithsonian Institution Press, Washington, USA. 409pp

Recibido: 2 de Febrero del 2017

Aceptado para publicación: 10 de Abril del 2017 
